\title{
The detection on tracking of a certain type equipment
}

\section{Based on AT89C52}

\author{
Kan Cheng ${ }^{1}$, Fu-Li Liu ${ }^{2}$, Wei Liu ${ }^{3}$ and Pei-Wen Liao ${ }^{4}$ \\ 1,2,3,4 Wuhan Mechanical Technology School,NO.1038,Luo Yu Road,Wuhan \\ City,Hubei Prov.China 430075 \\ ackokwd@mail.ustc.edu.cn, ${ }^{\mathrm{b}} 634824816 @ q q . c o m,{ }^{\text {cftk77165@aliyun.com, }{ }^{\mathrm{d}} 280870828 @ q q . c o m}$
}

Keywords: AT89C52、I/O、Tracking、Maintenance training

\begin{abstract}
For maintenance of a certain type of equipment, a detection Based on AT89C52 as the control center for the Certain Missile Equipment Tracking System was designed. The certain equipment elastic applies to all port resources of AT89C52,it complete power control, detection mode control,display driver, buzzer driver,motor driver. It realized operation training, principles display and maintenance in-one simulation training.
\end{abstract}

\section{Introduction}

With the steady progress of China's national defense construction, especially with the rapid development of equipment in recent years, requirements for equipment maintenance and support are also increasing,how to adapt to the new situation, close to actual combat becoming the major issue that armament support facing now. A certain type of equipment with tracking system has complex principle and higher stoppage rate that currently existing maintenance support equipment and maintenance can not be achieved using integrated simulation training. Based on all the facts, we proposed a certain type system with detection on tracking that can support state signal simulation, instructs the control logic and analog key signal.

System overall program. According to hydraulic system features of a certain type equipment and combined with requirements of intelligent, modular and convenience technical, we designed the overall system program as figure 1 shows.

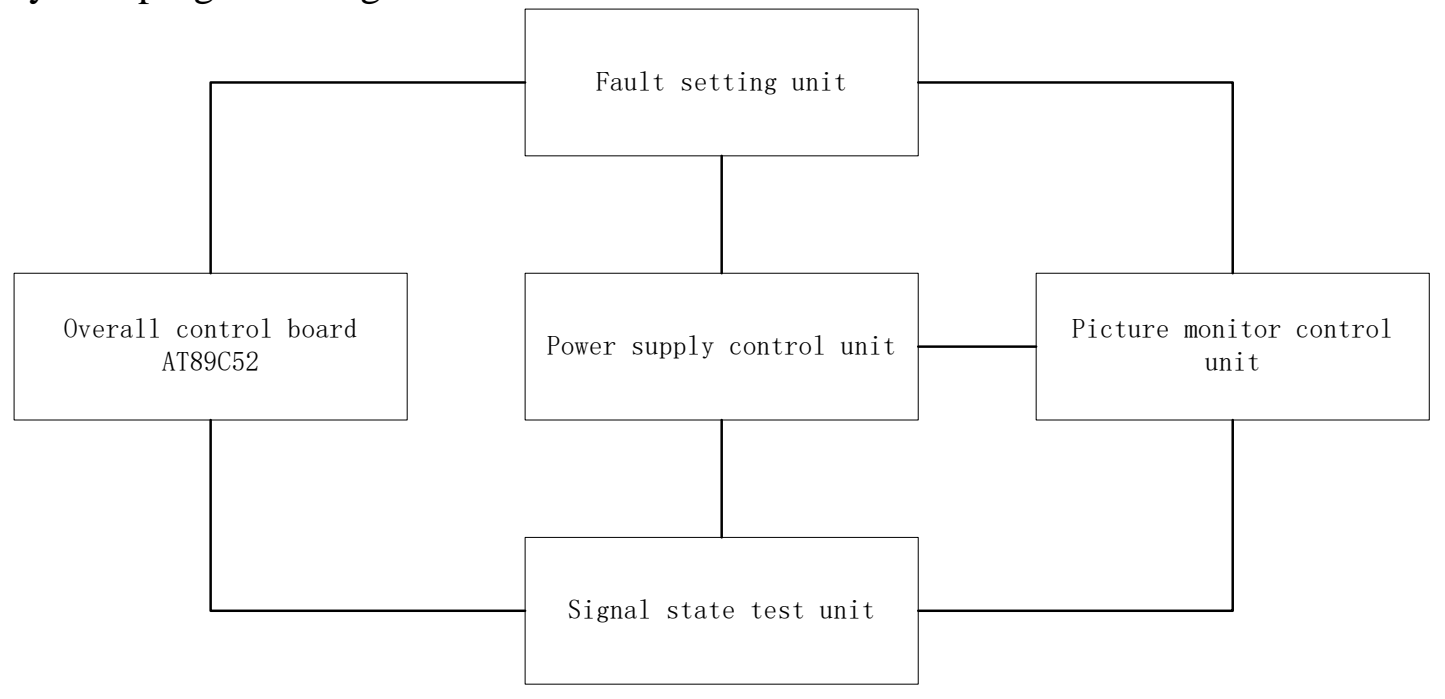

Figure 1 System overall architecture

System based on real detector simulation and fault repair that have simulationed mounting body frame unit, signal status unit, fault setting unit, status display unit. Mounting body frame simulation has guaranteed close to the equipment and have the same operating environment; Signal status unit 
keep port signal consistent with the real equipment and provide real-time test port to monitor mounting process status; Fault setting unit can reproduce mounting typical faults and test state point.Achieving channel conversion and logic control through relays and switching network.Status phenomenon analog display unit achieved by image display and LED,controlled by serial communication and PC software system to display operational flow state, the image data timely.Master core system is to achieve centralized control of each functional unit,to response to operator's Real-time operating state,to drive the corresponding functional units to implement the state pattern and process display.

Hardware design of the system. System control unit have external expansion functional module circuit with AT89C52 as the control core to collect digital and control the system. AT89C52 is a SCM of built-in eight central processing unit,it has 256bit RAM,8k bit ROM, 32-bit I/O ports, three 16 bit timer/counter, 5 two interrupts, and a full-duplex serial communication port.

As shown in Figure 2,power and reset circuit mainly uses MAX813 chip to monitor power troubleshooting and reset the device.Serial debug interface circuit mainly consists of MAX232 Low-Power RS-232 level converter chip and capacitance.Its function is to circuit Debugger AT89C52 SCM system.

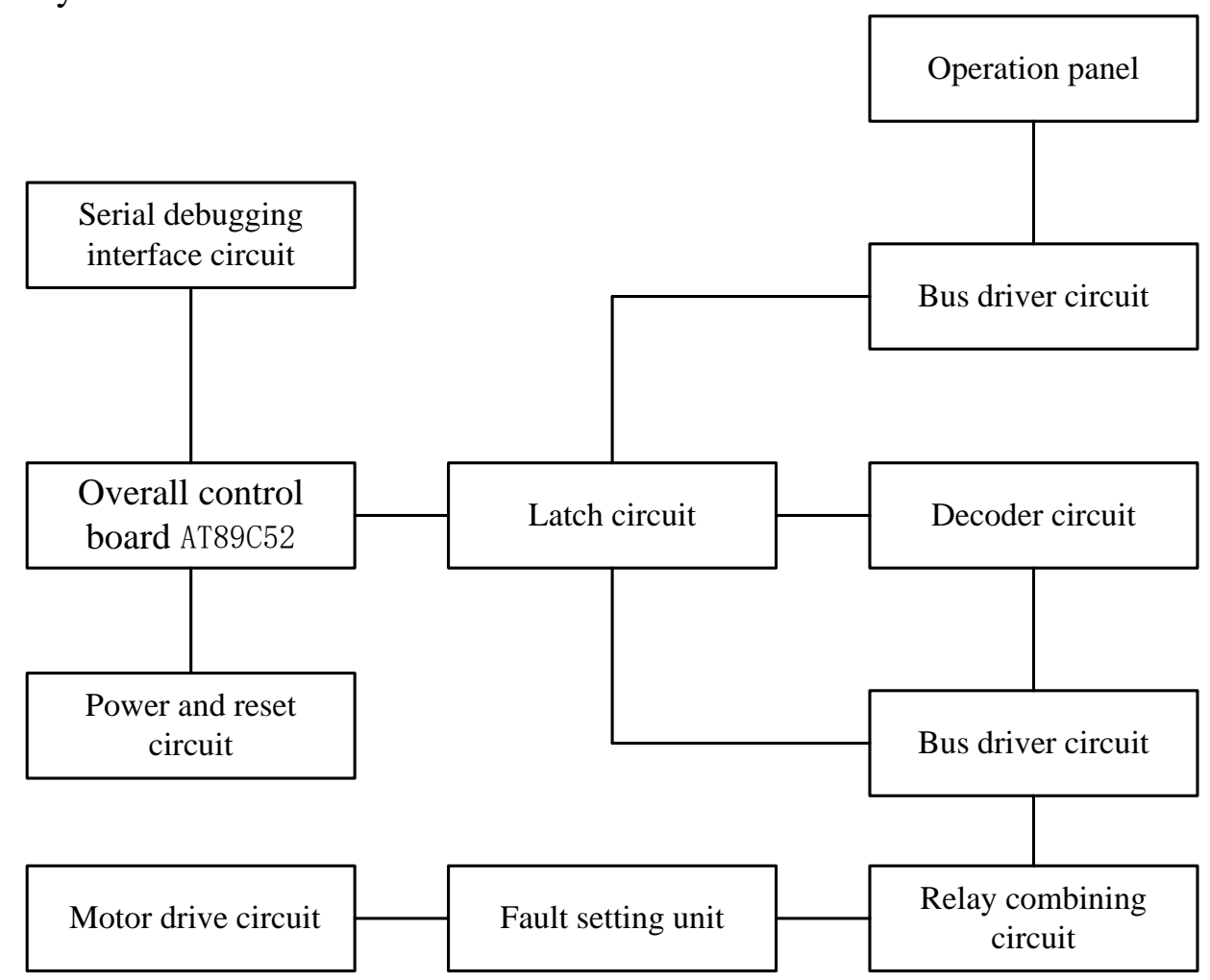

Figure 2 Block diagram of the MCU main control unit circuit configuration

Digital collection is through that the bus driver circuit receives(the main chip is 74HC244) the console interface and various switching command of the limit switch group.Feeding the I/O port of AT89C52 after the latch circuit(the main chip is octal tri-state non-inverting transparent latch 74HC573) processes.Setting the failure of limit switch set's various switches by cutting into fault signal through relay(HK4100F 24V relay) combining circuit.

The drive control of DC motor and solenoid valve is through the I/O port of AT89C52 sends control instructions,after latch handling, decoder circuit(the main chip is the 3-8 decoder 74HC138) selection and driving the bus driver circuit,Controlling solenoid valve or DC operation by relay combining circuit(reversed by hex inverter 74HC14,controlled by D822P transistor drive ,select turned by MY4NJ relay) finally. Failure settings of electromagnetic valve are through increasing the corresponding switches on control access links.

The system software design. system software divides into main control software systems, signal processing software system and PC software system . Transplant the C language code to SCM complete system control by keil program. 


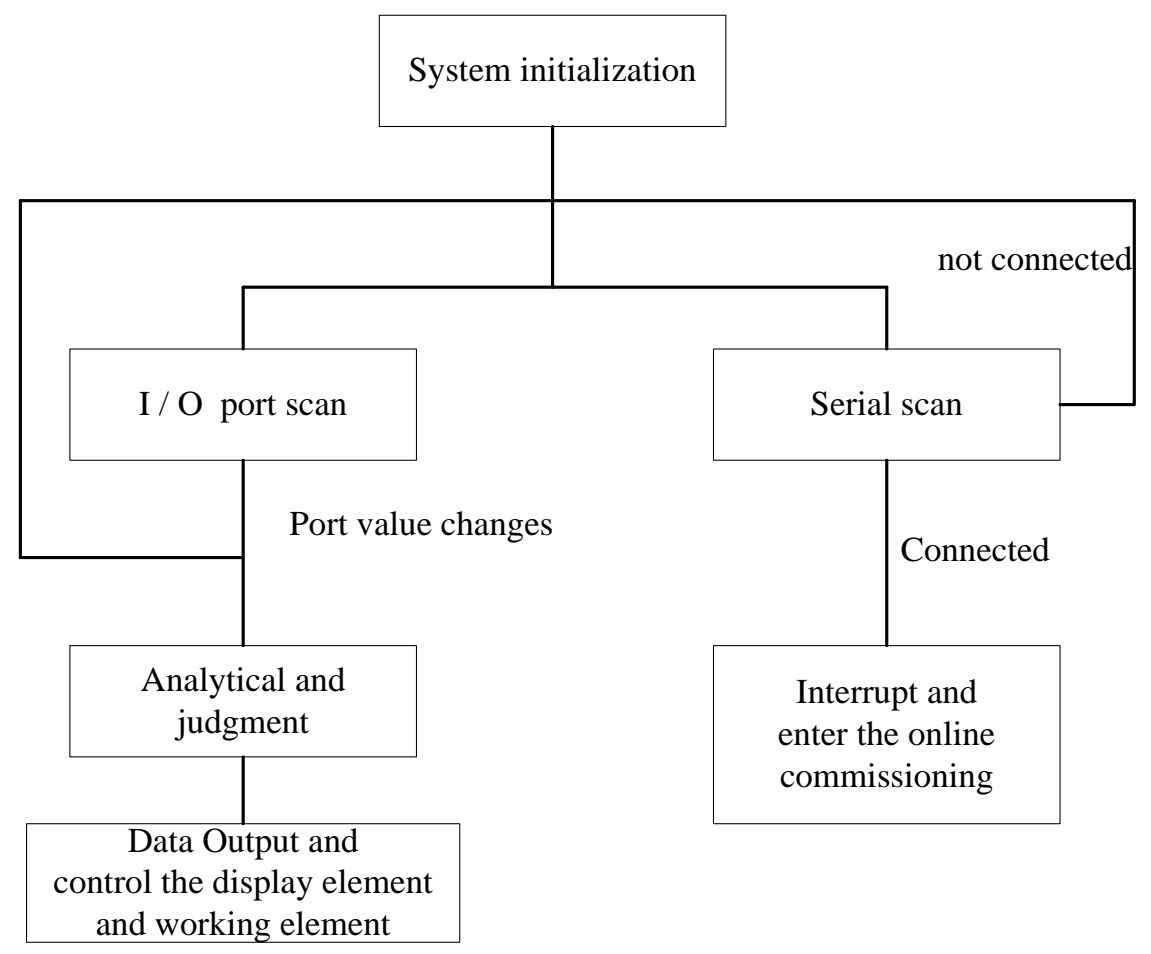

Figure 3 work process of Software system

Figure 3 is the process of master control software system,Entering the main program after system initialization.The main program scanned the port and $\mathrm{I} / \mathrm{O}$ port status at first,checked the serial connection state.If the connection is on,then entered the online commissioning state;Scanning I/O ports by the main program loop.If the connection is on that represents the PC software systems and master control program is connected properly. Then scanned status of each functional unit cyclically,in response to various state changes timely,achieved the driven display of state phenomenon .

\section{Conclusions}

The innovation of this article: firstly,it successfully applied embedded control, designed a certain type of missile equipment hydraulic system diagnostic equipment, Combined operation training and troubleshooting organically; Secondly, it set up a software system, completed panel operation and Accurate and reliable multi-machine serial communication channels, the software system is compact and efficient. Thirdly, the system is stable and reliable; man-machine interface is well, high reliability and maintainability, security and usability, its easy to operate.

\section{References}

[1] Gu Rongjiang and Zhang Xun, Establishment of GUI Software Development Platform Based on ARM,C hinese Jouranl of Liquid Crystals and Displays ,2009 24(6) , pp.603-857.

[2] Cheng Xijun and Tan Min, Serial Communication Technology in VC, Computer A pplications , 2001 21(9), pp.77-85.

[3] Ling Zhihao and Zhang Jianzheng, AT89C52 SCM principles and interface technology, 2011 26(9), pp.60-100.

[4] Stephen Prata, C Primer Plus, 2016 29(9), pp.60-100.

[5] Guo Tianxiang,51 singlechip and c language tutorial,2009, pp.30-80.

[6] Xia JiQiang ZMP, SCM experimental and practical tutorial.2001 2016 22(9).pp.56-93 . 
[7] Zhu FeiLong and Yang Ming. IAP function Remote Update System Based on Single Chip, Mechatronics Engineering, 2010 (09) 15-60.

[8] Zhou MaoXia,Windows API functions to achieve programming PC and microcontroller-based multi-machine communication, Shandong Normal University,2007(03)32-50.

[9] Chen Nan and so on. For the field of industrial and highly interconnected, TI launched a new Sitara ARM9 microprocessor. [J]. Global Electronics, 2010 (5) 86-87. 